Nicolai Spicher*, Markus Kukuk, Stefan Maderwald, and Mark E. Ladd

\title{
Multiscale Parameter Estimation (msPE) of QRS complexes distorted by magnetohydro- dynamic effects at 7 Tesla
}

https://doi.org/10.1515/cdbme-2019-0092

\begin{abstract}
During ultra-high-field magnetic resonance imaging, the acquisition of electrocardiography is impeded by artifacts. This poses a challenge in examinations where the QRS complex is used as a trigger for activating image acquisition. In this work, we customize a framework for multiscale parameter estimation for processing electrocardiography signals acquired inside and outside an ultra-high-field scanner bore: The parameters of a QRS complex modeled by a Gaussian function were estimated using scale-dependent algebraic expressions for 600 heartbeats from three subjects. Sensitivity $\geq 94 \%$ and accuracy $\leq 5 \mathrm{~ms}$ in each experiment indicate feasibility of this approach, and, additionally, run time is within the acceptable range for triggering latency in the majority of cases. Hence, we see a real-time implementation as an avenue for future work.
\end{abstract}

Keywords: Magnetic resonance imaging, Electrocardiography, Artifacts, Wavelet Transform, Parameter estimation

\section{Introduction}

Electrocardiography (ECG) is an established diagnostic method that allows acquisition of detailed information about a patient's cardiovascular health status. Acquisition during magnetic resonance imaging (MRI) is a long-standing problem due to susceptibility to noise [8]. Potential noise sources are magnetohydrodynamic (MHD) effects, radio-frequency pulses and activity of the gradient coils. At low field strengths, these effects can be compensated to a certain degree by making adjustments to the experimental setup, e.g. twisting cables [13]. However, these measures are no longer sufficient at ultra-highfield (UHF) MRI [6].

${ }^{\star}$ Corresponding author: Nicolai Spicher, Department of Computer Science, University of Applied Sciences and Arts Dortmund, Emil-Figge-Str. 42, 44227 Dortmund, Germany, e-mail: nicolai.spicher@fh-dortmund.de

Markus Kukuk, University of Applied Sciences and Arts

Dortmund, Dortmund, Germany.

Stefan Maderwald, Mark E. Ladd, Erwin L. Hahn Institute for Magnetic Resonance Imaging, Essen, Germany.

Mark E. Ladd, Deutsches Krebsforschungszentrum (German Cancer Research Center, DKFZ), Heidelberg, Germany. Medical Faculty, University of Duisburg-Essen, Essen, Germany
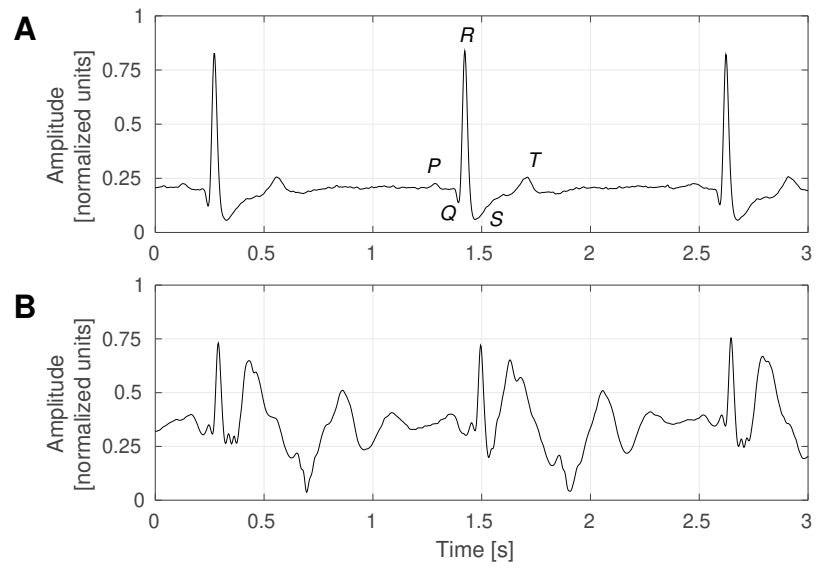

Fig. 1: ECG signals acquired A) outside and B) inside a 7T MRI bore from a healthy volunteer. Each heartbeat is represented by the sequence P-wave, QRS complex, and T-wave. The QRS complex represents the combination of Q-, R-, and S-wave.

Fig. 1 shows an ECG signal acquired outside and inside a UHF scanner bore. The latter is deformed by an increased amplitude of the T-wave, which is a typical MHD artifact [3]. Furthermore, the subtle Q- and S-waves have a reduced amplitude, resulting in a QRS complex similar to a Gaussian function. These artifacts are not only problematic for patient monitoring, where they can lead to false readings, but also when ECG is used for activation of image acquisition ("triggering"). The latency between occurrence of a QRS complex and trigger activation is critical, with a threshold of approx. 20ms [1].

In the past, several methods have been proposed for ECG triggering at UHF MRI, often applying a learning phase outside the scanner [12] or elaborate ECG hardware [4]; however, we aim to skip the learning phase and use a single lead from a basic physiological measurement unit (PMU) instead. The first is due to decreased examination time and the second is due to increased utility as a PMU is available at most sites.

Due to the basic ECG hardware, an algorithm robust to noise is required. In this work we perform an initial feasibility study for detecting QRS complexes in ECG segments and estimating their parameters using techniques from our recently proposed framework for multiscale parameter estimation (msPE) [5]. Our future aim is an algorithm processing an ECG signal from the PMU in real-time and triggering image acquisition each time a QRS complex is detected. 


\subsection{Related work}

Here, we give a short overview of algorithms for detecting QRS complexes in ECG signals acquired during MRI. An indepth review of these methods is given in [8]. Oster et al. proposed a QRS detector by applying the continuous wavelet transform (WT) to ECG signals and using heuristic rules for the processing of local maxima in the resulting scale-space [9].

Using a learning phase outside of the magnetic field, Gregory et al. proposed using a kernel compiled from 12-lead ECG measurement [2], and recently Stäb et al. were able to acquire MRI images successfully using a matched filter approach [12]. Additionally, Krug et al. proposed using independent component analysis [4], and Schmidt et al. proposed using non-linear filters and higher-order statistics [10].

Recently, we proposed the analytical msPE framework that makes use of a piecewise Gaussian derivative model [5].

\subsection{Mathematical background}

In this work, we use a customized implementation of msPE reduced to a minimum: As the Q- and S-wave of the QRS complex often vanish due to MHD noise, we use the scaledependent algebraic expressions for parameter estimation of a Gaussian function

$$
G_{\mu, \sigma, A, B}(t)=A \frac{1}{\sqrt{2 \pi} \sigma} \exp \left(-\frac{(t-\mu)^{2}}{2 \sigma^{2}}\right)+B
$$

instead of using the full Gaussian derivative model. As shown in Fig. 2A (left), $\mu$ represents the position, $A$ the amplitude, $B$ a constant baseline and $\sigma$ the width of the peak. We assume this signal is the input function $f(t)$ of the WT in form of a differential operator, proposed by Mallat [7]

$$
W T^{n} f(u, s)=s^{n} \frac{d^{n}}{d u}\left(f \star \theta_{s}\right)(u)
$$

where $s \in \mathbb{R}^{+}$is the scale, $n \in \mathbb{N}^{+}$denotes the number of derivatives taken and $\theta_{s}$ is a Gaussian-shaped wavelet. Hence, (2) is a multiscale representation of the input signal $f(t)$ that is convolved with Gaussians of different sizes. With increasing scale $s$, the width of the Gaussians increases, which results in a more intense smoothing effect. In [5] we showed that finding closed-form solutions for $n=1$ and $n=2$ results in three zero-crossings shown in Fig. 2 (B, left): The first order zero-crossing $\left(u 0_{0}^{1}(s)\right.$, black curve) represents the peak's local maximum and the two zero-crossings of the second order (left: $u 0_{-1}^{2}(s)$, right: $u 0_{+1}^{2}(s)$, gray curves) represent the two inflection points.

At the core of msPE lie equations for estimating the parameters of $G_{\mu, \sigma, A, B}$ from these zero-crossings. Therefore, in a nutshell, msPE reduces the problem of characterizing an input signal to the problem of selecting the correct

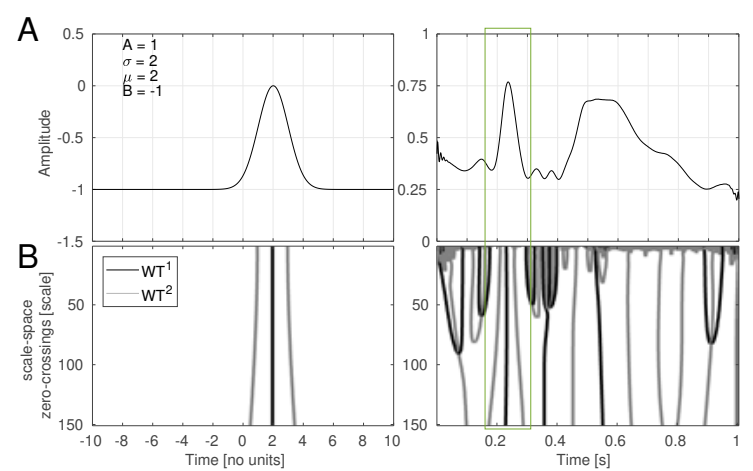

Fig. 2: A) Model (1) and ECG signal acquired during UHF MRI. B) Representation of signals as zero-crossings after application of WT operator (2) of order $n=1$ (black curves) and $n=2$ (gray curves). As can be seen, the model is a reasonable approximation of the QRS complex in both domains (green box).

zero-crossings from scale-space and substituting them into the equations. Using a suitable scale with low noise then allows estimation of the parameters with high accuracy.

Parameter $\mu$, representing the peak's center, can be obtained directly from the central zero-crossing:

$$
\mu(s)=u 0_{0}^{1}(s) .
$$

The peak's width is represented by parameter

$$
\sigma(s)= \pm \sqrt{\left(u 0_{*}^{2}(s)-\mu(s)\right)^{2}-\frac{s^{2}}{2}} .
$$

where $u 0_{*}^{2}(s)$ can be $u 0_{-1}^{2}(s)$ or $u 0_{+1}^{2}(s)$. Parameters $A$ and $B$ can not be computed from the scale-space representation (2) and therefore they are computed by using convolution $f_{c}(\mu, s)=\left(G_{\mu, \sigma, A, B} \star \theta_{s}\right)(u)$ :

$$
\begin{gathered}
B(s)=\frac{1}{\sqrt{\pi} \sqrt{s}}\left(\frac{f_{c}(\mu, s)-f_{c}(\mu-\sigma, s)}{\left.\exp \left(1 / s^{2}+2 \sigma^{2}\right) \sigma^{2}\right)-1}-f_{c}(\mu-\sigma, s)\right) \\
A(s)=-\frac{\sqrt{\frac{2 \sigma^{2}}{s}+s}\left(\sqrt{\pi} B \sqrt{s}+f_{c}(\mu, s)\right)}{\sqrt{2 \pi} \sigma} .
\end{gathered}
$$

$G_{\mu, \sigma, A, B}$ is only a special case of the more general Gaussian derivative model and a step-by-step derivation of the model parameters is provided in [5].

However, as can be seen from Figs. 1 and 2, the QRS complex is not symmetric as is $G_{\mu, \sigma, A, B}$. Hence, we introduce a piecewise model by splitting the model at the center $\mu$ :

$$
G_{\mathrm{pw}}(t)= \begin{cases}G_{A, \sigma_{\mathrm{L}}, \mu, B_{\mathrm{L}}}(t), & \text { for } t<\mu \\ G_{\bar{A}, \sigma_{\mathrm{R}}, \mu, B_{\mathrm{R}}}(t), & \text { for } t \geq \mu,\end{cases}
$$

where $\bar{A}=B_{\mathrm{L}}+A-B_{\mathrm{R}}$ to make sure that there is a seamless transition between both sides. This approach allows computation of the parameters of the model's left/right side by substituting the zero-crossings of the $u 0_{-1}^{2}(s) / u 0_{+1}^{2}(s)$ into the equations. 


\section{Materials and Methods}

\subsection{Study data}

We re-analyzed a subset of data from a previous study [11]: ECG signals (400 $\mathrm{Hz}$ sampling rate) were acquired using the probe provided by the MRI vendor (MAGNETOM 7T; Siemens GmbH, Erlangen, Germany) connected wirelessly to the PMU. Data were acquired from three volunteers $(27-33$ years) within two experiments, the first with the patient table in home position $(\approx 0.3 \mathrm{~T})$ and the second with the table in the iso-center (7T). MR imaging was not performed, to ensure that only MHD effects degrade ECG signals. For each experiment, 100 ECG segments (400ms) containing a QRS complex were delineated manually and, as ground truth, the point in time when the QRS complex reaches its maximum was stored.

\subsection{Algorithm}

We propose a basic algorithm taking advantage of properties shown in Fig. 2: I) Scale-space zero-crossings associated with model features form uninterrupted lines. Hence, the simple algorithmic task of detecting these lines allows for extracting all zero-crossings potentially associated with the model. II) The central $W T^{1}$ line of the model is straight, which allows for detecting the correct one from all found $W T^{1}$ lines. III) The smoothing effect increases with scale. This allows for using higher scales when substituting zero-crossings into the equations for increased robustness during parameter estimation.

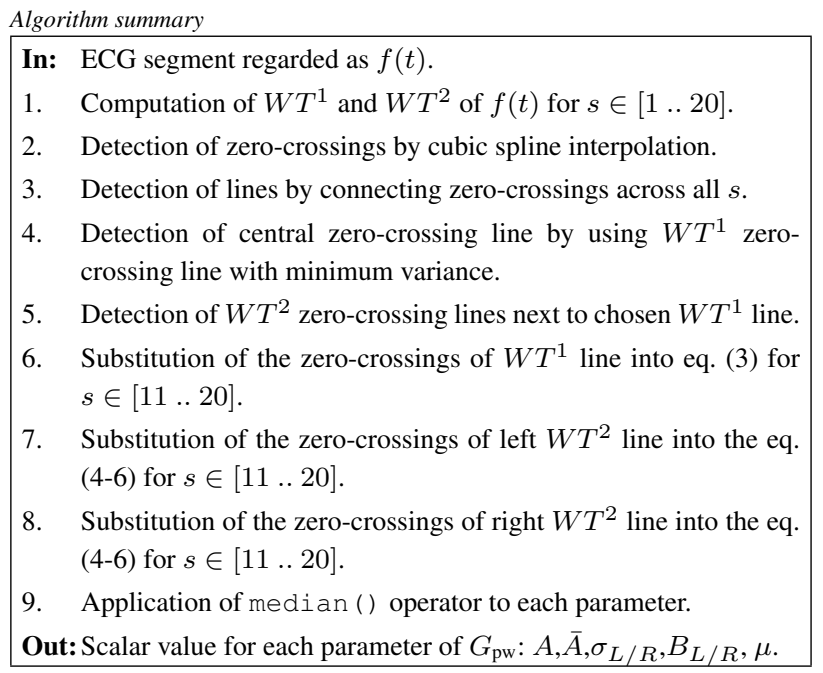

\subsection{Evaluation methodology}

Three measures for evaluating feasibility were computed: For each processed ECG segment, the run time was stored.
Tab. 1: Results of experiments. Accuracy and processing times are given as arithmetic mean \pm standard deviation (s.d.). Fig. 3 shows corresponding visualizations for subjects I-III at 7 Tesla.

\begin{tabular}{lcccc}
\hline Subj. & Tesla & Sensitivity [\%] & Accuracy [ms] & Time [ms] \\
\hline I & 0.3 & 99.0 & $11.5 \pm 1.8$ & $17.5 \pm 3.4$ \\
II & 0.3 & 94.0 & $3.4 \pm 2.0$ & $16.4 \pm 2.0$ \\
III & 0.3 & 98.0 & $-4.4 \pm 2.6$ & $15.4 \pm 2.5$ \\
\multicolumn{2}{l}{ Average } & $\mathbf{9 7 . 0}$ & $\mathbf{3 . 5} \pm \mathbf{2 . 2}$ & $\mathbf{1 6 . 4} \pm \mathbf{2 . 6}$ \\
\hline I & 7.0 & 95.0 & $-2.3 \pm 2.7$ & $17.4 \pm 3.0$ \\
II & 7.0 & 97.0 & $8.4 \pm 4.8$ & $16.4 \pm 2.0$ \\
III & 7.0 & 95.0 & $7.8 \pm 2.9$ & $17.5 \pm 2.6$ \\
\multicolumn{2}{l}{ Average } & $\mathbf{9 5 . 7}$ & $\mathbf{4 . 6} \pm \mathbf{3 . 5}$ & $\mathbf{1 7 . 1} \pm \mathbf{2 . 6}$ \\
\hline
\end{tabular}

Furthermore, accuracy was estimated by measuring the difference between the computed position $\mu$ and the ground truth. If it was larger than $\pm 50 \mathrm{~ms}$, the QRS complex was considered missed. Sensitivity was defined as the percentage of detected QRS complexes to all 100 within an experiment.

\section{Results}

Tab. 1 shows the results averaged for each experiment: As can be seen, the sensitivity is $\geq 94 \%$ for all experiments and averaged mean accuracy is below $\leq 5 \mathrm{~ms}$. At $7 \mathrm{~T}$, results are only slightly degraded (mean: $+1.1 \mathrm{~ms}$ s.d.: $+1.3 \mathrm{~ms}$ ). Fig. 3 shows signals from these experiments: As can be seen, the estimated curves are similar to the shapes of the actual QRS complex, if the correct central zero-crossing line $u 0_{0}^{1}$ is chosen (green curves). If a wrong $u 0_{0}^{1}$ is chosen, results are severely wrong (magenta curve, indicated by arrow).

An off-the-shelf laptop was used for data processing. Computation of a single ECG segment took below $20 \mathrm{~ms}$ on average, but $\pm 2 \times$ s.d. is above $20 \mathrm{~ms}$.

\section{Conclusion}

In this work, we proposed an algorithm for detecting QRS complexes and estimating their parameters in ECG segments biased by MHD effects. This work is a feasibility study for our future aim of triggering MRI image acquisition in real-time. Using data from three volunteers shows that sensitivity and accuracy were only slightly downgraded due to MHD noise.

Higher sensitivity has been reported in some related work (e.g. 99.1\% [4]), but elaborate ECG hardware, which is not available at all MR sites, was used (e.g. 12-lead ECG in [4]). Our accuracy is better than values reported in [4] (5.8 $\pm 5.0 \mathrm{~ms})$, but our small sample size and the omittance of aspects that 

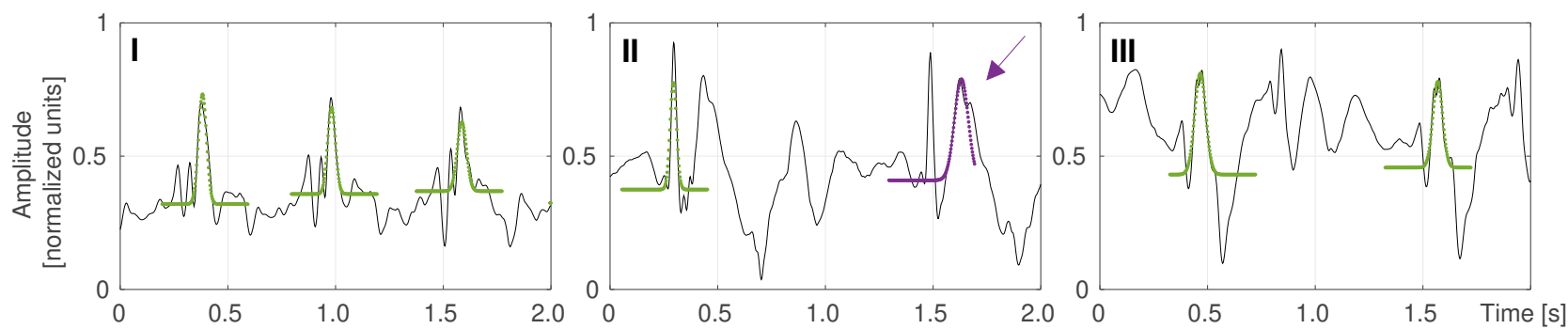

Fig. 3: Example results from all subjects at UHF MRI. The QRS complexes are very similar to a Gaussian (subject I), or in proximity to the T-wave with high amplitude (II), or are followed by a large undershoot (III). The estimated parameters are substituted into the model function (1) and visualized as green curves when the displacement from the ground truth is within $\pm 50 \mathrm{~ms}$ or magenta curves otherwise.

would be required for a real-time trigger algorithm, make it unclear weather the same performance can be expected for actual MRI triggering within a large cohort. Computation time was in $\approx 68 \%$ of cases below the proposed latency for errorfree MR image acquisition [1].

In future work, run time could be decreased by using fewer scales, but this requires an in-depth analysis of scale range vs. accuracy. Missed peaks in most cases result from a wrongly chosen center line (e.g. Fig. 3 magenta curve). Apparently, using the the straightness of a line is not a robust criterion in the presence of MHD noise, indicating the need for a more elaborate line classification for improved sensitivity.

In summary, we belive that the results of this feasibility study warrant the development of a real-time implementation of msPE for UHF MRI triggering in future work.

\section{Author Statement}

Research funding: The authors state no funding involved. Conflict of interest: Authors state no conflict of interest. Informed consent: Informed consent has been obtained from all individuals included in this study. Ethical approval: The research related to human use complies with all the relevant national regulations, institutional policies and was performed in accordance with the tenets of the Helsinki Declaration, and has been approved by the authors' institutional review board or equivalent committee.

\section{References}

[1] Fischer, S. E., Wickline, S. A., And Lorenz, C. H. Novel real-time R-wave detection algorithm based on the vectorcardiogram for accurate gated magnetic resonance acquisitions. Magnetic resonance in medicine 42 (Aug. 1999), 361-370.

[2] Gregory, T. S., Schmidt, E. J., Zhang, S. H., And Tse, Z. T. H. 3DQRS: A method to obtain reliable QRS complex detection within high field MRI using 12-lead electrocardiogram traces. Magnetic Resonance in Medicine 71, 4 (jan 2014), 1374-1380.

[3] Gupta, A., Weeks, A., And Richie, S. Simulation of Elevated T-Waves of an ECG Inside a Static Magnetic Field
(MRI). IEEE Transactions on Biomedical Engineering 55, 7 (jul 2008), 1890-1896.

[4] Krug, J. W., Rose, G., Clifford, G. D., And Oster, J. ECG-based gating in ultra high field cardiovascular magnetic resonance using an independent component analysis approach. Journal of Cardiovascular Magnetic Resonance 15, 1 (nov 2013).

[5] Kunuk, M., AND SPICher, N. Parameter estimation based on scale-dependent algebraic expressions and scale-space fitting. IEEE Transactions on Signal Processing 67, 6 (mar 2019), 1431-1446.

[6] Ladd, M. E., Bachert, P., Meyerspeer, M., Moser, E., Nagel, A. M., Norris, D. G., Schmitter, S., Speck, O., StRAUB, S., AND ZAISS, M. Pros and cons of ultra-highfield MRI/MRS for human application. Progress in Nuclear Magnetic Resonance Spectroscopy 109 (dec 2018), 1-50.

[7] Mallat, S. Zero-crossings of a wavelet transform. IEEE Transactions on Information Theory 37, 4 (jul 1991), 10191033.

[8] Oster, J., AND Clifford, G. D. Acquisition of electrocardiogram signals during magnetic resonance imaging. Physiological Measurement 38, 7 (jun 2017), R119-R142.

[9] Oster, J., Pietquin, O., Abacherli, R., Kraemer, M., AND FELBLINGER, J. A specific QRS detector for electrocardiography during MRI: Using wavelets and local regularity characterization. In 2009 IEEE International Conference on Acoustics, Speech and Signal Processing (apr 2009), IEEE.

[10] Schmidt, M., Krug, J. W., Rosenheimer, M. N., AND Rose, G. Filtering of ECG signals distorted by magnetic field gradients during MRI using non-linear filters and higher-order statistics. Biomedical Engineering / Biomedizinische Technik 63, 4 (jul 2018), 395-406.

[11] Spicher, N., Kukuk, M., Maderwald, S., And Ladd, M. E. Initial evaluation of prospective cardiac triggering using photoplethysmography signals recorded with a video camera compared to pulse oximetry and electrocardiography at $7 \mathrm{~T}$ MRI. BioMedical Engineering OnLine 15, 1 (nov 2016).

[12] StÄB, D., Roessler, J., O'Brien, K., Hamilton-Craig, C., AND BARTH, M. ECG Triggering in Ultra-High Field Cardiovascular MRI. Tomography (Ann Arbor, Mich.) 2 (Sept. 2016), 167-174.

[13] Wendt, R. E., Rokey, R., Vick, G. W., AND Johnston, D. L. Electrocardiographic gating and monitoring in NMR imaging. Magnetic Resonance Imaging 6, 1 (jan 1988), 8995. 